\title{
RELATIVITÉ D'ÉCHELLE ET MORPHOGENÈSE
}

\author{
Laurent NotTALE
}

RÉsumé : La théorie de la relativité d'échelle se propose de généraliser les lois de la mécanique à un mouvement continu mais non-différentiable. Cela implique trois conséquences au moins : a) l'espace-temps et donc ses géodésiques deviennent fractals; b) il existe une infinité de géodésiques entre deux points, ce qui conduit à une description non-déterministe; c) le concept de vitesse se dédouble, ce qu'on traduit par un formalisme complexe. L'équation fondamentale de la dynamique s'intègre alors sous forme d'une équation de Schrödinger, dont les solutions définissent des densités de probabilité qui peuvent s'interpréter en termes de morphologies naturellement hiérarchisées. Cette approche théorique conduit également à proposer des généralisations aux lois invariantes d'échelle, susceptibles d'applications dans des domaines autres que la physique.

Mots-CLÉs : relativité, échelles, fractales, structures, évolution, phénomènes critiques.

ABSTRACT: The theory of scale relativity attempts to generalize the laws of mechanics to continuous but non-differentiable motion. This implies at least three consequences : a) the space-time and its geodesics become fractal; b) there are an infinity of geodesics between any two points, which leads to a non-deterministic description; $c$ ) the concept of velocity becomes two-valued, which we account for in terms of a complex formalism. Then the fundamental equation of dynamics can be integrated under the form of a Schrödinger equation. Its solutions define probability densities which we interpret in terms of naturally hierarchized morphologies. This theoretical approach also leads to suggest some generalizations of scale invariant laws, which could be applied in domains other than physics.

KEYWORDS : relativity, scales, fractals, structures, evolution, critical phenomena. 
ZuSAMMENFASSUNG: In der Theorie der Skalen-Relativität wird versucht, die Gesetze der Mechanik so zu verallgemeinern, daß sie auf kontinuierliche, aber nicht differenzierbare Bewegungen anwendbar sind. Daraus ergeben sich mindestens drei Konsequenzen : a) die Raumzeit und ihre Geodäsie werden zu Fraktalen; b) zwischen zwei Punkten gibt es unendlich viele geodätische Linien, woraus sich eine nicht-deterministische Beschreibung ergibt; c) der Begriff der Geschwindigkeit wird doppeldeutig, was in einem komplexen Formalismus zum Ausdruck kommt. Die Grundgleichung der Dynamik wird dann in Gestalt einer Schrödinger-Gleichung integriert, durch deren Lösungen Wahrscheinlichkeitsdichten definiert werden, die als von Natur aus hierarchisch angeordnete Morphologien verstanden werden können. Dieser theoretische Ansatz führt zu Verallgemeinerungen skalarinvarianter Gesetze, die möglicherweise auch in Gebieten außerhalb der Physik angewandt werden können.

STICHWÖRTER : Relativität, Maßstäbe, Fraktale, Strukturen, Evolution, kritische Phänomene.

RIASSUNTO : La teoria di scala propone di generalizzare le leggi della meccanica ad un movimento continuo ma non differenziabile. Ciò implica almeno tre conseguenze : a) lo spazio-tempo e quindi le sue geodetiche diventano frattali; b) esistono un infinità di geodetiche tra due punti, il che porta ad una descrizione non determinista; $c)$ il concetto di velocità si raddoppia, il che viene interpretato con $i$ numeri complessi. L'equazione fondamentale della dinamica si integra quindi sotto forma di un'equazione di Schrödinger, le cui soluzioni definiscono delle densità di probabilità che possono interpretarsi come morfologie naturalmente gerarchizzate. Questo approccio teorico porta anche a proporre delle generalizzazioni delle leggi invarianti di scala, suscettibili di essere applicate in campi diversi dalla fisica.

PAROLE CHIAVE : relatività, scale, frattali, strutture, evoluzione, fenomeni critici.

Laurent NotTale, né en 1952, est directeur de recherche au Centre national de la recherche scientifique, à l'observatoire de Paris-Meudon. Il est spécialiste d'astrophysique et de physique théorique.

Adresse : DAEC/CNRS, Observatoire de Meudon, F-92195 Meudon Cedex.

Courrier électronique : laurent.nottale@obspm.fr 
Dans le domaine de l'astrophysique extragalactique et de la cosmologie, un des principaux problèmes posé actuellement est celui de l'existence de structures hiérarchisées sur une large gamme d'échelles. Un enjeu important de cette science est ainsi la construction d'une théorie de la formation et de l'évolution des grandes structures de l'univers.

Il est remarquable de rencontrer, dans d'autres champs que ceux de la physique, qu'on pourrait penser si différents a priori, en particulier dans le domaine biologique, le même type de problèmes et de préoccupations. Qu'on pense simplement à la structure hiérarchisée des organismes vivants complexes: ADN dans le noyau, noyau dans la cellule, cellule dans le tissu, tissu dans l'organe, organe dans l'organisme. La complexité est explicite ici à travers l'existence de niveaux d'organisation imbriqués, chaque niveau étant caractérisé par l'existence d'une taille caractéristique, souvent même matérialisée dans le cas du vivant par des parois ou frontières. Cette hiérarchie de structures se poursuit d'ailleurs au niveau de l'organisation sociale et politique.

Voici, par exemple, certains des thèmes débattus lors d'un récent colloque d'écologie marine ou halieutique ${ }^{1}$ :

«La prise en compte de la dimension spatiale des phénomènes est une des grandes préoccupations actuelles de l'ensemble des sciences halieutiques. » «Les hétérogénéités spatiales concernent en effet tous les aspects du système pêche. »

«Étudier la diversité spatiale, son hétérogénéité et sa structuration, [...] s'avère ainsi de plus en plus incontournable pour analyser et comprendre la dynamique des systèmes halieutiques[...]»

« Du banc au stock et à l'écosystème : quelles dynamiques à quelles échelles? Quelles échelles pertinentes pour la recherche halieutique?»

«Du local au global [...]»

La physique est depuis longtemps confrontée au problème de la répartition spatiale très inhomogène de la matière dans l'univers. Cette répartition des structures spatiales est souvent hiérarchisée, que ce soit dans le domaine microscopique (quarks dans les nucléons, nucléons dans le noyau, noyau et électrons dans l'atome, atome dans la molécule...) ou macroscopique (étoiles et leur système planétaire formant des amas, se regroupant avec la matière interstellaire, elle-même fractale, en galaxies, celles-ci formant à leur tour des paires, des groupes et des amas, qui appartiennent à

1. BEz, 2000. 
des superamas de galaxies, eux-mêmes sous-ensembles des très grandes structures de l'univers). Ce qui frappe dans ces deux cas est que c'est le vide et non la matière qui domine, même à de très grandes échelles où l'on pensait auparavant trouver une distribution homogène.

La théorie de la relativité d'échelle ${ }^{2}$ a été construite précisément pour traiter des questions de structuration en échelle. On y prend en compte une intervention explicite des échelles d'observation (ce qui revient à travailler dans le cadre d'une géométrie fractale), ou plus généralement de celles qui sont caractéristiques des phénomènes étudiés, ainsi que des relations entre ces échelles, par l'introduction d'un espace des résolutions. Une description de structures à toutes les échelles (ou sur une large gamme) induit une dynamique nouvelle dont le comportement devient du type quantique plutôt que classique. Or, comme nous allons le voir à travers quelques exemples, une telle dynamique conduit naturellement à une morphogenèse, étant génératrice de structures organisées de manière hiérarchique, en rapport avec les conditions extérieures (forces et conditions aux limites). Un des acquis d'une telle approche théorique par rapport au problème posé est, en particulier, qu'elle génère des rayons caractéristiques « quantifiés » pour les structures ainsi constituées. De plus, la prise en compte de lois d'échelle non-linéaires, plus générales que celles des fractals self-similaires, permet de mettre en évidence des comportements nouveaux qui pourraient servir de modèles à certaines structures biologiques.

\section{DYNAMIQUE INDUITE}

On est conduit, en relativité d'échelle, à généraliser une nouvelle fois le concept d'espace-temps et à travailler dans le cadre d'un espace-temps fractal $^{3}$. On prend alors en compte des systèmes de coordonnées (et des trajectoires, en particulier les géodésiques de l'espace fractal) eux-mêmes fractals, c'est-à-dire possédant une structure interne. Nous ne nous concentrerons pas, dans cette section, sur les descriptions possibles d'une telle structure, qui porte sur l'espace des échelles ${ }^{4}$, mais plutôt sur ses effets induits sur les déplacements dans l'espace ordinaire. La combinaison entre eux de ces effets conduit à l'introduction d'un outil de description du type de celui de la mécanique quantique (mais avec une interprétation différente). On y abandonne la description classique en terme de conditions

2. Nottale, 1989, 1992 et 1993.

3. Ord, 1983; Nottale et Schneider, 1984; Nottale, 1989 et 1993 ; El Naschie, 1995.

4. Nottale, 1992,1993 et 1996 a. 
initiales et de trajectoires individuelles déterministes, au bénéfice d'une description statistique en terme d'amplitudes de probabilité.

\section{1. Équation de Schrödinger généralisée}

Rappelons l'essence de la méthode utilisée dans le cadre de la relativité d'échelle pour passer d'une dynamique classique à une dynamique de type quantique.

Les trois conditions (elles-mêmes déductibles de l'abandon de l'hypothèse de différentiabilité) qui permettent de transformer l'équation fondamentale de la dynamique en une équation de Schrödinger sont les suivantes :

a) il existe un très grand nombre (à la limite, une infinité) de trajectoires potentielles;

b) chacune de ces trajectoires est une courbe fractale; on peut considérer successivement les cas : (i) d'une dimension fractale constante ${ }^{5} D=2$, qui correspond par exemple au mouvement brownien; (ii) puis d'autres valeurs de la dimension ${ }^{6}$; (iii) d'une dimension variable, devenue la composante d'un vecteur dans le cadre des transformations d'échelle loglorentziennes de la relativité d'échelle restreinte ${ }^{7}$; (iv) d'une dimension variable dans le cadre de transformations non-linéaires incluant une «dynamique d'échelle ${ }^{8} »$;

c) il y a irréversibilité au niveau infinitésimal, c'est-à-dire non-invariance dans la réflexion de l'élément différentiel de temps $d t \rightarrow-d t$.

La première condition conduit à adopter une description de type «fluide », où l'on ne considérera plus seulement la vitesse d'une trajectoire individuelle, mais le champ de vitesse $v[x(t), t]$ de toutes les trajectoires virtuelles.

La deuxième condition mène à décomposer les déplacements élémentaires sous forme d'une moyenne, $d x=v d t$, et d'une fluctuation $d \xi$, caractérisée par une loi de comportement fractale, $d \xi \propto d t^{1 / D}$.

La troisième condition a pour conséquence un dédoublement des vitesses, y compris au niveau des vitesses moyennes et, plus généralement, un dédoublement de l'opérateur de dérivation par rapport au temps.

On est ainsi amené à introduire un double processus tridimensionnel

$$
d X_{ \pm}^{i}=d x_{ \pm}^{i}+d \xi_{ \pm}^{i},
$$

dans lequel $d x_{ \pm}^{i}=v_{ \pm}^{i} d t,<d \xi_{ \pm}^{i}>=0$ et

\footnotetext{
5. Nottale, 1993.

6. Nottale, 1995 et 1996 a.

7. Nottale, 1992.

8. Nottale, $1997 \mathrm{~b}$.
} 


$$
<\frac{d \xi_{ \pm}^{i}}{d t} \frac{d \xi^{j}}{d t}>= \pm \delta^{i j}\left(\frac{2 \mathcal{D}}{d t}\right)^{2-2 / D} .
$$

(On a fait ici $c=1$ pour simplifier l'écriture; $\delta^{i j}$ représente le symbole de Kronecker). $\mathcal{D}$ est un paramètre d'échelle fondamental qui caractérise le comportement fractal des trajectoires. Ce paramètre détermine la transition essentielle qui apparaît dans un tel processus entre le comportement fractal à petite échelle (où les fluctuations dominent) et non fractal à grande échelle (où le mouvement classique moyen redevient dominant). Le paramètre $D$ dans l'exposant est la dimension fractale. Seule la dimension $D=$ 2 sera examinée ici $^{9}$.

Une représentation simple du dédoublement de variables dû à l'irréversibilité consiste en l'utilisation de nombres complexes. On définit un opérateur complexe de dérivation par rapport au temps,

$$
\frac{d}{d t}=\frac{1}{2}\left(\frac{d_{+}+d_{-}}{d t}-i \frac{d_{+}-d_{-}}{d t}\right),
$$

puis une vitesse moyenne complexe qui résulte de l'action de cet opérateur sur la variable de position :

$$
V^{i}=\frac{d}{d t} x^{i}=V^{i}-i U^{i}=\frac{v_{+}^{i}+v^{i}}{2}-\mathrm{i} \frac{v_{+}^{i}-v^{i}}{2} .
$$

Ainsi, à la limite classique où $v_{+}^{i}=v^{i}$, la partie réelle de cette vitesse complexe s'identifie à la vitesse classique tandis que la partie imaginaire disparaît.

Après avoir défini les lois des déplacements élémentaires dans un tel processus fractal et localement irréversible, il nous faut maintenant analyser les effets de ces déplacements sur d'autres grandeurs physiques. Considérons une fonction $\mathrm{f}(X(t), t)$. Sa dérivée totale par rapport au temps s'écrit :

$$
\frac{d \mathrm{f}}{d t}=\frac{\partial \mathrm{f}}{\partial t}+\nabla \text { f. } \frac{d \mathrm{X}}{d t}+\frac{1}{2} \frac{\partial^{2} \mathrm{f}}{\partial X_{i} \partial X_{i}} \frac{d X_{i} d X_{i}}{d t} .
$$

On peut alors calculer les dérivées « avant » et « arrière » de f. Dans ce calcul, la valeur moyenne de $d X / d t$ devient $d_{ \pm} x / d t=v_{ \pm}$, tandis que $\left\langle d X_{i} d X_{j}>\right.$ se réduit à $\left\langle d \xi_{ \pm i} d \xi_{ \pm j}>\right.$, si bien que le dernier terme de l'équation (5) se transforme en un Laplacien du fait de l'équation (2). On obtient alors :

$$
d_{ \pm} \mathrm{f} / d t=\left(\partial / \partial t+v_{ \pm} \cdot \nabla \pm \mathcal{D} \Delta\right) \mathrm{f} .
$$

En combinant enfin ces deux dérivées, on obtient l'expression de l'opérateur complexe de dérivation par rapport au temps :

9. Voir Nottale, 1995 et 1996a, pour une analyse des effets des écarts à cette dimension critique. 


$$
\frac{d}{d t}=\frac{\partial}{\partial t}+V \cdot \nabla-i \mathcal{D} \Delta .
$$

Il contient deux termes imaginaires supplémentaires, $-i U$. $\nabla$ et $i \mathcal{D} \Delta$, en plus de l'opérateur de dérivation totale ordinaire, $d / d t=\partial / \partial t+V . \nabla$.

Nous pouvons maintenant réécrire l'équation fondamentale de la dynamique en utilisant cet opérateur de dérivation : celle-ci prendra alors automatiquement en compte les nouveaux effets considérés. On obtient :

$$
m \frac{d^{2}}{d t^{2}} x=-\nabla \phi
$$

où $\phi$ est un terme de potentiel.

Plus généralement, on peut reprendre le déroulement de la construction de la mécanique lagrangienne avec ce nouvel outil ${ }^{10}$. Le caractère complexe de la vitesse $V$ implique celui de la fonction de Lagrange, donc de l'action $\mathcal{S}$.

Une fonction d'onde $\Psi$ s'introduit alors très simplement comme une réexpression de cette action complexe :

$$
\Psi=e^{i s / 2 \mathrm{~m} \mathcal{D}} .
$$

Elle est reliée à la vitesse complexe comme suit :

$$
V=-2 i \mathcal{D} \nabla(\ln \Psi) \text {. }
$$

Nous pouvons maintenant changer d'outil de description et écrire l'équation d'Euler-Newton (8) en fonction de cette fonction d'onde :

$$
2 i m \mathcal{D} \frac{d}{d t}(\nabla \ln \Psi)=\nabla \phi \text {. }
$$

Après quelques calculs, cette équation s'intègre sous forme d'une équation de Schrödinger :

$$
\mathcal{D}^{2} \Delta \Psi+i \mathcal{D} \frac{\partial}{\partial t} \Psi-\frac{\phi}{2 m} \Psi=0 .
$$

On retrouverait l'équation de la mécanique quantique standard par le choix $\mathcal{D}=\hbar / 2 m$. En posant $\Psi \Psi^{*}=\rho$, on trouve que la partie imaginaire de cette équation est l'équation de continuité,

$$
\partial \rho / \partial t+\operatorname{div}(\rho V)=0
$$

ce qui justifie l'interprétation de $\rho$ comme une densité de probabilité.

\subsection{Application à la gravitation}

Dans le cas où l'on applique cette approche au problème des structures gravitationnelles macroscopiques (voir $\S 1.4$ ), il est remarquable que

10. Nottale, 1993, 1996a et 1997a. 
l'équation (8), qui combine effets quasi-quantiques et gravitationnels, peut se mettre sous forme d'une équation du mouvement inertiel dans le vide. En effet, l'équation de Newton du mouvement dans un champ gravitationnel est la limite newtonienne de l'équation des géodésiques de la théorie d'Einstein, qui s'écrit via la dérivée covariante de la relativité générale comme :

$$
0=D u^{u} / d s=d u^{u} / d s+\Gamma^{\mu}{ }_{v \rho} u^{v} u^{\rho} .
$$

À la limite newtonienne ${ }^{11}$, le potentiel gravitationnel $\varphi$ est lié au coefficient temporel de la métrique par la relation $g_{00}=1+2 \varphi$ (on fait $c=1$ pour simplifier l'écriture). En fonction du potentiel défini dans le paragraphe précédent, on a $\varphi=\varnothing / \mathrm{m}$. Toujours à cette limite, on a également $u^{0} \approx 1$, si bien que la composante temporelle de cette équation est négligeable, que $d s=d t$, et que seuls subsistent les symboles de Christoffels $\Gamma^{\alpha}{ }_{00}$ $=\partial^{\alpha} \varphi$, avec $\alpha=1,2,3$. Par conséquent, l'équation (14) devient :

$$
0=D u^{\alpha} / d t=d u^{\alpha} / d t+\Gamma_{00}^{\alpha}\left(u^{0}\right)^{2}=d u^{\alpha} / d t+\partial^{\alpha} \varphi .
$$

On peut alors définir un opérateur de dérivation covariante $\emptyset / d t$ combinant les effets de courbure et de non-différentiabilité et s'appliquant à la vitesse devenue complexe. Ainsi l'équation du mouvement localement inertiel dans le vide, dépourvu de toute force, écrite en terme de cet opérateur :

$$
\frac{D}{d t} V^{\alpha}=0 \Rightarrow \frac{d}{d t} V^{\alpha}+\Gamma_{00}^{\alpha}=\frac{d}{d t} V^{\alpha}+\partial^{\alpha} \varphi=0,
$$

s'intègre sous forme de l'équation de Newton-Schrödinger (12), qui mélange des effets quasi-quantiques et gravitationnels.

\subsection{Modèle hiérarchique}

Le processus décrit dans les paragraphes précédents fournit naturellement une structuration hiérarchisée. En effet, l'équation de Schrödinger généralisée ci-dessus s'applique à la distribution de probabilité des positions de «particules » préalablement définies. Mais ce concept de «particule » consiste ici précisément à décrire le mouvement et la répartition d'objets ou de groupes d'objets indépendamment de leur structure interne. Le résultat obtenu (décrit par la densité de probabilité) est que de telles particules se regroupent elles-mêmes en des structures. Autrement dit, elles forment un nouvel objet à une échelle supérieure. Dans un changement d'échelle, le nouvel objet peut être pris comme «particule», et si les

11. Voir, p. ex., Landau et Lifchitz, 1970. 
conditions de base dont nous sommes partis restent respectées (grand nombre de trajectoires virtuelles, caractère fractal de chaque trajectoire, irréversibilité), une nouvelle équation de Schrödinger généralisée pourra lui être appliquée, cela impliquant la formation d'un nouveau niveau hiérarchique. Il faut remarquer qu'il n'y aura pas forcément self-similarité stricte entre ces niveaux, car les conditions aux limites et/ou la forme des potentiels appliqués auront pu changer dans le changement d'échelle.

\subsection{Application : oscillateur harmonique}

Comme exemple d'application de cette méthode, le potentiel en $r^{2}$ d'un oscillateur harmonique (c'est-à-dire d'une force proportionnelle à $r$ ) est particulièrement instructif. En cosmologie, il correspond à la situation de la formation de structures à partir d'un milieu de densité constante, situation qui n'est pas structurante en cosmologie classique mais le devient dans la nouvelle approche. Plus généralement, ce type de potentiel apparaît naturellement dans toutes les situations de petits écarts par rapport à une position d'équilibre, et est donc une bonne première approximation pour de nombreux problèmes.

Considérons le cas d'une densité moyenne égale à $\rho$. (Dans une autre situation, on remplacera simplement dans l'équation ci-après $\pi G \rho / 3$ par une constante adaptée au problème posé). On obtient l'équation de Schrödinger suivante :

$$
\mathcal{D}^{2} \Delta \Psi+i \mathcal{D} \frac{\partial}{\partial t} \Psi-\frac{\pi}{3} G \rho r^{2} \Psi=0
$$

Nous allons rechercher les solutions stationnaires de cette équation. L'équation indépendante du temps s'écrit :

$$
\mathcal{D}^{2} \Delta \Psi+\left[\frac{E}{2 m}-\frac{\pi}{3} G \rho r^{2}\right] \Psi=0 .
$$

À la substitution près $h \rightarrow 2 m \mathcal{D}$, c'est l'équation de Schrödinger de la mécanique quantique standard pour un oscillateur harmonique tridimensionnel isotrope de fréquence

$$
\omega=\left(\frac{4 \pi G \rho}{3}\right)^{1 / 2} .
$$

Le rapport de l'énergie sur la masse est quantifié comme :

$$
E / m=4 \mathcal{D}\left(\frac{\pi G \rho}{3}\right)^{1 / 2}\left(n+\frac{3}{2}\right),
$$


où $n=n_{1}+n_{2}+n_{3}$, les $n_{\mathrm{i}}$ correspondant à trois oscillateurs linéaires pour les trois coordonnées. La densité de probabilité de l'état stationnaire s'écrit :

$$
\left|\Psi_{n 1 n 2 n 3}\right|^{2} \propto e^{-(r / a)^{2}}\left[\mathcal{H}_{n 1}(x / a) \mathcal{H}_{n 2}(y / a) \mathcal{H}_{n 3}(z / a)\right]^{2},
$$

où les $\mathcal{H}_{n}$ 's sont les polynômes d'Hermite, et où $a$ est une échelle de longueur caractéristique donnée par:

$$
a=\sqrt{2 \mathcal{D} / \omega}=(\mathcal{D})^{1 / 2}(\pi G \rho / 3)^{-1 / 4} .
$$

On rappelle que les premiers polynômes d'Hermite sont :

$$
\mathcal{H}_{0}=1 ; \mathcal{H}_{1}=2 x ; \mathcal{H}_{2}=4 x^{2}-2 ; \mathcal{H}_{3}=8 x^{3}-12 x ; \ldots
$$

On prédit donc une formation de structures suivant les divers modes de l'oscillateur harmonique quantifié (voir figure 1). Le mode zéro est une gaussienne de dispersion $\sigma_{0}=a / a \sqrt{2}$. Le mode $n=1$ est une structure double dont les pics sont situés en $x_{\text {pic }}= \pm a$. Le mode $n=2$ est dégénéré en deux types de structures, une structure linéaire à trois pics en $0, \pm \sqrt{5 / 2} a \approx$ $\pm 1.58 a$ et une structure en carré. Pour $n=3$, on trouve $x_{\text {pic }}= \pm 0.602 a$ et $\pm 2.034 a$ pour la structure linéaire, etc.

Il faut noter que Raphaël Hermann (1997) a montré par ailleurs qu'une simulation numérique des équations de la dynamique écrites sous leur forme «classique » (équation 10) générait bien les solutions de l'équation de Schrödinger ${ }^{12}$. On voit effectivement sur la figure 1 que les particules s'organisent en structures simples $(n=0)$, doubles $(n=1)$, multiples (chaîne triple ou quadrilatère pour $n=2$ ), etc., c'est-à-dire déjà sur deux niveaux de hiérarchie. Il est remarquable que de telles structures se rencontrent précisément dans les zones de formation d'étoiles ainsi que dans les groupes compacts de galaxies, que certains auteurs considèrent comme des galaxies en formation.

Ainsi, l'exemple de l'oscillateur harmonique démontre clairement comment cette dynamique nouvelle ayant un caractère quasiquantique (en laquelle s'est transformée la dynamique classique du fait de la nondifférentiabilité) est naturellement génératrice d'une morphogenèse de type hiérarchique. Un autre exemple est fourni par le potentiel central képlérien : appliqué au système solaire, pour lequel le potentiel du Soleil est dominant, ce cas permet d'expliquer sa structuration en distance, dont on peut montrer qu'elle est organisée en au moins cinq niveaux hiérarchiques successifs auto-similaires les uns par rapport aux autres ${ }^{13}$. Il a également permis de prévoir théoriquement des structures maintenant observées dans les systèmes planétaires extra-solaires récemment découverts ${ }^{14}$.

12. Hermann, 1997.

13. Nottale, Schumacher et Gay, 1997; Schumacher et Nottale, 2001.

14. Nottale, 1996b; Nottale, Schumacher et Lefèvre, 2000. 


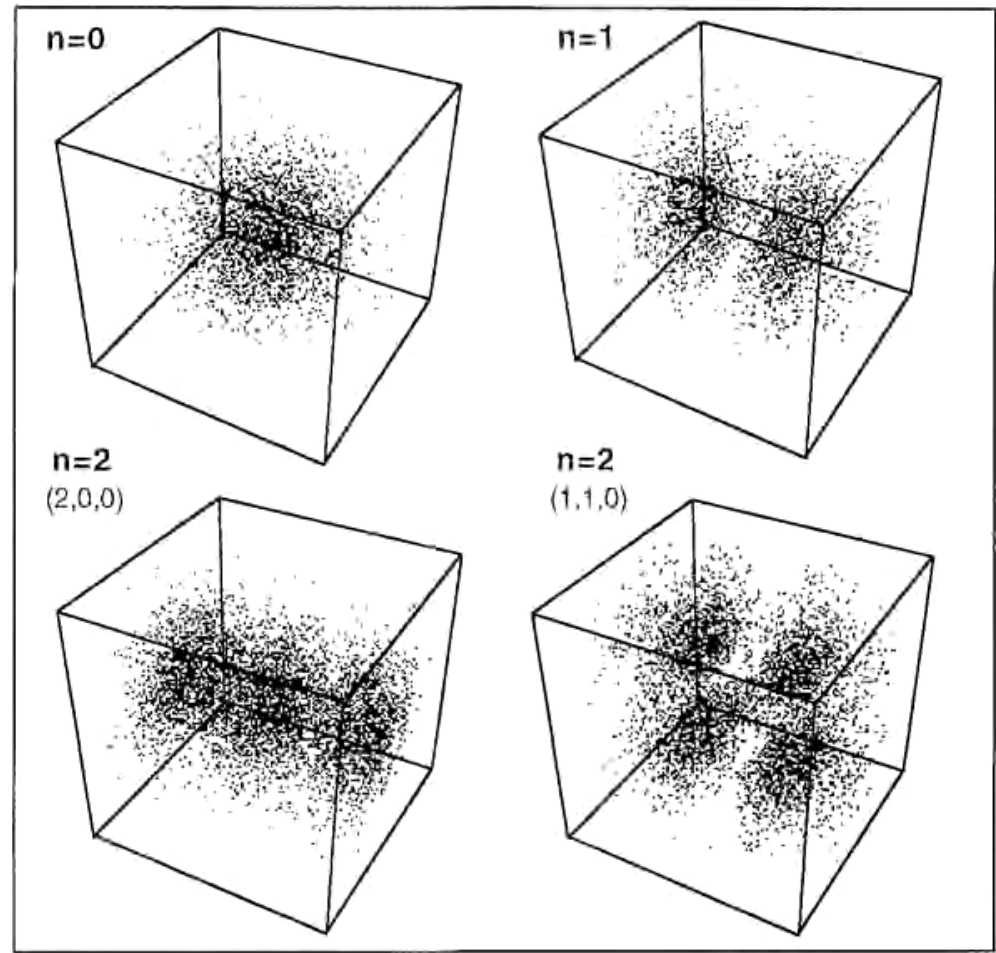

Fig. 1. - Représentation (en densité de points) des solutions de l'équation de Schrödinger (équation 18) pour le potentiel de l'oscillateur harmonique tridimensionnel isotrope. Pour obtenir cette figure, on a jeté des particules au hasard dans la densité de probabilité donnée par le carré du module de l'amplitude de probabilité $\Psi$. On voit que les particules s'organisent naturellement en amas et ensembles d'amas.

Il reste bien sûr à vérifier si une telle méthode est applicable dans des domaines autres que ceux de la physique. Cette méthode consiste à construire une théorie de la morphogenèse de structures allant au-delà de la description des trajectoires individuelles des «particules » qui forment ces structures. Il semble qu'il en soit bien ainsi, par exemple, en halieutique pour les poissons de haute mer, qui se regroupent effectivement en banc, les bancs en amas, les amas en population ${ }^{15}$ : que tel ou tel individu soit dans tel banc, que tel ou tel banc participe de tel amas, etc. n'a pas

15. BEZ, 2000. 
d'importance en soi du point de vue de la structure. De plus, les trois hypothèses dont nous sommes partis (infinité de trajectoires potentielles, chaque trajectoire est fractale, elles sont localement irréversibles) semblent bien adaptées au comportement d'espèces marines. La structuration obtenue comme solution d'équations de Schrödinger généralisées (existence de pics bien marqués de la densité de probabilité, hiérarchie de structures) semble en bon accord, au moins qualitatif pour le moment, avec ce comportement observé.

\section{LOIS D’ÉCHELLE GÉNÉRALISÉES}

Les effets induits sur les déplacements et le mouvement décrits dans les paragraphes précédents sont une manifestation de la plus simple des lois d'échelle possibles (laquelle intervient dans la description de la fluctuation fractale): une loi de puissance correspondant à une dimension fractale constante $D=2$. Dans une transformation d'échelle, une telle loi possède la structure mathématique du groupe de Galilée, et satisfait donc, de manière triviale, au principe de relativité d'échelle.

L'analogie avec le mouvement peut être poussée plus loin. On sait, d'une part, que le groupe de Galilée n'est qu'une approximation du groupe de Lorentz (correspondant à la limite $c \rightarrow \infty$ ), d'autre part, que tous deux restent une description d'un comportement inertiel, alors que c'est avec la dynamique que la physique du mouvement trouve toute sa complexité.

Il en est de même du point de vue des lois d'échelle. Les fractals de dimension constante sont l'équivalent pour les échelles de ce qu'est l'inertie galiléenne pour le mouvement. On peut alors proposer de généraliser les lois de dilatation et de contraction usuelles de deux manières :

a) La première consiste à introduire un groupe de Lorentz de transformation d'échelle ${ }^{16}$. Il y apparaît une échelle de résolution finie, minimale ou maximale, invariante sous les dilatations, qui remplace le zéro ou l'infini tout en gardant les propriétés physiques de ceux-ci (voir aussi mon article, supra, p. 11-25). Nous avons proposé d'identifier ces échelles respectivement à la longueur de Planck et à l'échelle de la constante cosmologique. Cette situation correspond cependant toujours à une transformation d'échelle linéaire sur les résolutions.

b) La seconde consiste à prendre en compte des transformations d'échelle non-linéaires, c'est-à-dire à passer à une «dynamique

16. Nottale, 1992. 
d'échelle ${ }^{17}$ » et, si possible, à une relativité généralisée d'échelle. Ce sont des exemples de telles lois généralisées que nous allons considérer dans ce qui suit.

\subsection{Invariance d'échelle discrète et comportement fractal log-périodique : "onde d'échelle »}

\subsubsection{Lois log-périodiques}

Parmi les corrections à l'invariance d'échelle (caractérisée par des lois de puissance), l'une d'entre elles est amenée à jouer un rôle potentiellement important dans de nombreux domaines qui ne se réduisent pas à la seule physique. Il s'agit des lois log-périodiques, qui peuvent être définies par l'apparition d'exposants ou de dimensions fractales complexes.

Didier Sornette et ses collaborateurs ${ }^{18}$ ont montré qu'un tel comportement fournit une description satisfaisante et potentiellement prédictive de nombreux phénomènes de crise temporelle, incluant tremblements de terre et krachs boursiers. Jean Chaline, Laurent Nottale et Pierre Grou ${ }^{19}$ ont utilisé de telles lois d'échelle comme modèle de la chronologie des sauts évolutifs majeurs dans l'évolution des espèces ${ }^{20}$. Ils ont montré qu'elles s'appliquaient aussi à la chronologie des grandes crises économiques depuis le Néolithique ${ }^{21}$. Il s'agit là d'une première étape vers la description d'un type de comportement de crises temporelles qui pourrait se révéler très général.

Nous allons montrer ici qu'on peut obtenir une correction log-périodique à une loi de puissance à partir de l'exigence de covariance d'échelle ${ }^{22}$, c'est-à-dire de conservation de la forme des équations dépendantes d'échelle ${ }^{23}$.

Considérons une variable explicitement dépendante de la résolution, $\Phi(\varepsilon)$. Dans les applications considérées, la variable $\varepsilon$ sera identifiée à l'intervalle de temps $\left|T-T_{\mathrm{c}}\right|$, où $T_{\mathrm{c}}$ est la date critique. Supposons que $\Phi$ satisfasse à une équation différentielle du premier ordre du type de celles du groupe de renormalisation,

$$
\frac{d \Phi}{d \ln \varepsilon}-D \Phi=0
$$

17. NotTale, $1997 \mathrm{~b}$.

18. Voir Sornette, 1997, 1998, et les références citées dans ces textes.

19. Chaline, Nottale et Grou, 1999.

20. Grou, Nottale et Chaline, 2001.

21. Voir, pour plus de détails, Nottale, Chaline et Grou, 2000.

22. Nottale, 1989.

23. Voir, aussi, Pocheau, 1997. 
dont la solution est une loi de puissance, $\Phi(\varepsilon) \propto \varepsilon^{D}$.

Dans la recherche de corrections d'ordre suivant à cette loi, on remarquera qu'introduire directement un exposant complexe n'est pas satisfaisant: cela conduirait à des fluctuations log-périodiques de grande ampleur plutôt qu'à une correction à la loi de puissance pouvant rester petite. Nous allons donc, dans une deuxième étape, faire l'hypothèse que l'annulation de la différence (équation 24) n'était qu'approximative, et que le deuxième membre de cette équation est en fait non nul :

$$
\frac{d \Phi}{d \ln \varepsilon}-D \Phi=\chi .
$$

Nous exigerons alors que la nouvelle fonction $\chi$ soit solution d'une équation qui garde la même forme que l'équation initiale :

$$
\frac{d \chi}{d \ln \varepsilon}-D^{\prime} \chi=0
$$

En posant $D^{\prime}=D+\delta$, on trouve alors que $\Phi$ est solution d'une équation générale du deuxième ordre

$$
\frac{d^{2} \Phi}{(d \ln \varepsilon)^{2}}-B \frac{d \Phi}{d \ln \varepsilon}+C \Phi=0 .
$$

où $B=2 D+\delta$ et $C=D(D+\delta)$. Cette solution s'écrit $\Phi(\varepsilon)=a \varepsilon^{D}(1+b$ $\left.\varepsilon^{\delta}\right)$, où $b$ peut maintenant être arbitrairement petit. Finalement, le choix d'un exposant imaginaire $\delta=i \omega$ mène à une solution dont la partie réelle inclut une correction log-périodique :

$$
\Phi(\varepsilon)=a \varepsilon^{D}[1+b \cos (\omega \ln \varepsilon)] .
$$

Des fluctuations log-périodiques ont également été obtenues dans le cadre relativiste d'échelle à travers la ré-interprétation de l'invariance de jauge et de la nature de l'électromagnétisme qui peut y être proposée ${ }^{24}$. Rappelons brièvement comment.

Dans l'idée de décrire un couplage entre comportement en échelle et mouvement, on considère des résolutions (supposées décrire la structure de l'électron en deçà de son échelle de Compton) qui deviennent dépendantes de la position et de l'instant. On trouve alors que l'on peut identifier la fonction « arbitraire » qui intervient dans l'invariance de jauge à un rapport de résolutions, ce qui permet d'écrire la fonction d'onde de l'électron sous la forme (à une constante près dans la phase)

$$
\Psi=\Psi_{0} e^{i 4 \pi \alpha \ln \varepsilon}
$$

qui décrit ainsi une onde dans l'espace des résolutions. Sa partie réelle prend bien la forme $\log$-périodique précédente, $\cos (\omega \ln \varepsilon)$, où $\omega$ s'identifie

24. Nottale, 1996a. 
maintenant à la constante de structure fine (c'est-à-dire au carré de la charge électrique) à un facteur numérique près. Dans le cas des lois de dilatation log-lorentziennes de la relativité d'échelle restreinte, les rapports de résolutions possible à l'intérieur (des structures) de l'électron deviennent limités par le rapport entre son échelle de Compton et l'échelle de Planck. Cela implique la quantification de la charge et l'existence d'une relation entre sa masse et sa charge, qui s'écrit en première approximation (8 $\alpha / 3)$ $\ln \left(m_{\mathrm{p}} / m_{\mathrm{e}}\right)=1$, dans laquelle $\alpha$ est la valeur de la constante de structure fine à basse énergie, $m_{\mathrm{p}}$ est la masse de Planck et $m_{\mathrm{e}}$ la masse de l'électron. Le facteur (8/3) est issu de la théorie électrofaible. Cette relation est vérifiée à $0,3 \%$ par les données expérimentales et à $0,01 \%$ en tenant compte des corrections liées aux effets de seuil ${ }^{25}$.

\subsubsection{Applications : évolution des espèces et crises économiques}

Apparues par des sauts brusques du patrimoine génétique, les espèces biologiques peuvent évoluer graduellement ou rester en stase pendant de longues périodes et s'éteindre individuellement ou à l'occasion de crises d'extinction en masse ${ }^{26}$. Les sauts entre les espèces sont à l'origine de bifurcations qui permettent d'assimiler le schéma évolutif global à un arbre de la biodiversité, «l'arbre de la vie». Ainsi les noeuds de cet arbre peuvent être identifiés aux grands sauts évolutifs, et les « longueurs » des branches aux intervalles de temps entre ces événements majeurs.

Chaline, Nottale et Grou ${ }^{27}$ ont récemment proposé de comparer la chronologie de ces sauts pour un certain nombre de lignées évolutives à un modèle d'accélération ou de décélération log-périodique. Il s'agit d'un modèle à deux paramètres, dépendant d'une époque critique de convergence $T_{c}$ (ou de divergence s'il s'agit d'une décélération) et du rapport d'échelle $g$ entre intervalles de temps successifs. Une date de la séquence considérée s'écrit en fonction de son rang $n$ sous la forme : $T_{n}=T_{c}+\left(T_{0}-\right.$ $\left.T_{c}\right) g^{-n}$, où $T_{0}$ est un événement quelconque de la lignée.

On trouve une accélération log-périodique statistiquement significative pour l'évolution globale de la vie depuis la première cellule, pour les équidés d'Amérique du Nord, pour les dinosaures sauropodes, pour l'ordre des rongeurs (après une décélération initiale possible), ainsi que pour les primates incluant les homininae. En ce qui concerne les échinodermes, on trouve une décélération à partir de leur date d'apparition. Les différentes lignées sont caractérisées, comme on peut s'y attendre, par des époques critiques différentes (voir figure 2, p. 108).

25. Gould et Eldredge, 1977.

26. Chaline, Nottale et Grou, 1999.

27. Chaline, Nottale et Grou, 1999. 


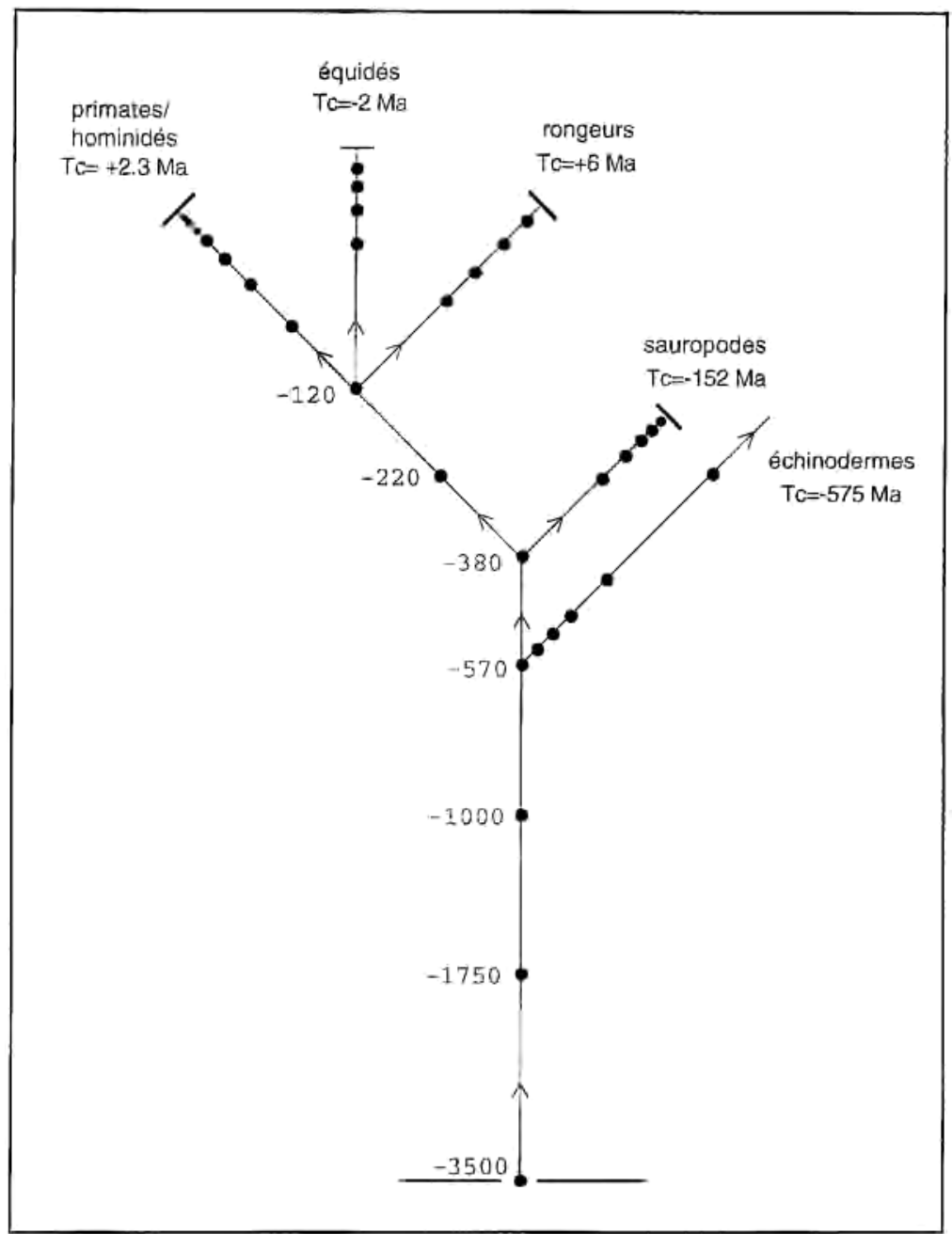

Fig. 2. - Dans cette figure sont reportées les dates des événements marquants de: a) l'histoire "globale» de la vie; b) des dinosaures sauropodes; c) des trois lignées de mammifères étudiées à titre d'exemple, rongeurs, primates et équidés; d) des échinodermes. Les temps sont comptés le long des branches (et non pas suivant l'ordonnée de la figure), en échelle logarithmique suivant $\log \left(\mathrm{T}_{0}-\mathrm{T}\right)$, où l'on a pris comme point zéro $\mathrm{T}_{0}=+100$ millions d'années dans le futur. Ce choix permet de visualiser les accélérations et les temps critiques différents de chaque lignée. Les valeurs reportées pour les époques critiques $\mathrm{T}_{c}$ sont ajustées pour chaque lignée en incluant tous les ancêtres jusqu'à l'origine de la vie (sauf en ce qui concerne les échinodermes, qui montrent une décélération à partir de leur date d'apparition à -570 Ma). 
Il est remarquable, que, pour toutes les lignées étudiées, la valeur de $T_{c}$, qu'on peut espérer être un paramètre intrinsèque à la lignée, ne dépend pas des seuils adoptés pour le choix des dates (c'est-à-dire du fait qu'on n'inclut que les événements majeurs principaux, ou également des événements de moindre ampleur). Par contre, la valeur de $g$ n'a bien sûr rien d'intrinsèque, car elle dépend de la densité des dates choisie dans l'espace des échelles ln $\varepsilon$ (ainsi, doubler par exemple le nombre de dates remplace $g$ par sa racine). Dans les cas d'accélérations, nous avons proposé d'interpréter ces dates critiques comme la fin de la capacité d'évolution de la lignée considérée.

Le même type de loi rendait compte de la chronologie des grandes crises économiques depuis le Néolithique ${ }^{28}$ (voir figure 3 ci-dessous). On trouve, dans ce cas, une époque critique située à la fin du xxi ${ }^{\mathrm{e}}$ siècle, $T_{\mathrm{c}}=2080 \pm 30$.
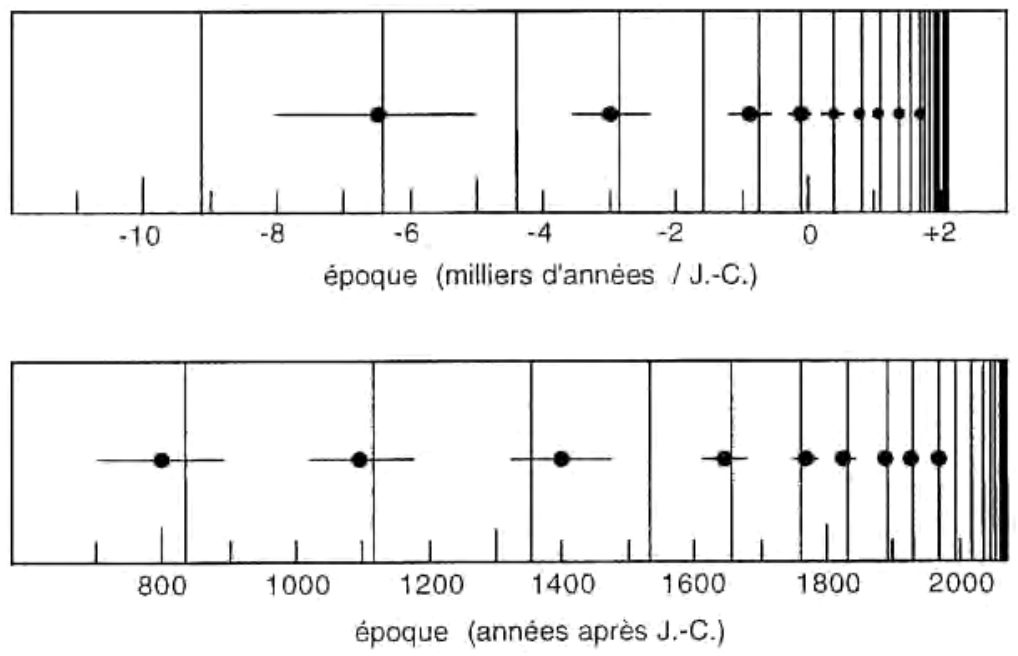

Fig. 3. - Comparaison entre une loi log-périodique à base $\mathrm{g}=1.32$ et $\mathrm{T}_{\mathrm{c}}=2078$, et les périodes de grandes crises économiques depuis le Néolithique identifiées par Grou $^{29}$. Le point donne l'époque médiane de la crise et la barre son intervalle de durée totale; l'absence de barre indique que la durée de la crise est de l'ordre de la taille du point (voir alors le diagramme suivant à plus petite échelle). Les prochaines crises sont prédites : à l'époque récente (1995-2000), en 2015-2020, puis 2030-2035, etc., toutes ces dates participant de la "super-crise » culminant en $2080 \pm 30$.

28. Nottale, Chaline et Grou, 2000.

29. Grou, 1987. 
Remarquons, pour finir, que ce type de loi, sachant qu'il s'applique aussi bien à des phénomènes physiques tels que les tremblements de terre ou des fractures dans des matériaux, ne nous renseigne probablement pas sur les mécanismes de l'évolution des espèces ou des sociétés. Ce n'est pas une loi spécifique des phénomènes biologiques, mais sans doute une contrainte physique qui s'applique également à eux, à partir du moment où il y a évolution, mais indépendamment de sa cause. On peut faire la conjecture que l'apparition de lois fractales caractéristique des phénomènes critiques est liée au fait qu'il s'agit d'un processus à mémoire, chaque niveau d'organisation nouveau prenant appui sur les précédents (ainsi les noyaux apparaissent dans des cellules, puis les multicellulaires apparaissent constitués de cellules à noyaux, puis des exosquelettes pour ces multicellulaires, etc.).

\subsection{Force d'échelle}

Considérons maintenant le cas de la «dynamique d'échelle ». Comme nous l'indiquions dans ce qui précède, le comportement strictement invariant d'échelle à dimension fractale constante correspond à un comportement libre du point de vue de la physique d'échelle. Aussi, de même qu'il existe des forces qui impliquent un écart au mouvement inertiel, on s'attend également à voir les systèmes fractals naturels présenter des distorsions par rapport au comportement autosimilaire. Par analogie, de telles distorsions peuvent, dans une première étape, être attribuées à l'effet d'une « force d'échelle » ou encore d'un «champ d'échelle».

Avant d'introduire ce concept, rappelons le renversement qui doit être effectué en ce qui concerne les variables d'échelle (voir aussi mon article, supra p. 11-25). Considérons une courbe fractale dont la longueur dépend explicitement de la résolution comme $\mathcal{L}=\mathcal{L}_{0}(\lambda / \varepsilon)^{\delta}$. On définit en général la dimension d'échelle comme

$$
\delta=\frac{d \ln \mathcal{L}}{d \ln (\lambda \varepsilon)} .
$$

Mais, dans le but de passer à une relativité d'échelle généralisée, on est amené à redéfinir $\delta$ comme une variable, qui joue pour les échelles le rôle joué par le temps en ce qui concerne le mouvement, et à définir la résolution à partir de $\ln \mathcal{L}$ et $\delta$ :

$$
V=\ln (\lambda \varepsilon)=\frac{d \ln \mathcal{L}}{d \delta} .
$$

On peut alors faire l'hypothèse que les lois d'échelle peuvent être construites à partir d'un principe de moindre action. On introduit une fonction de Lagrange d'échelle, $V(\ln \mathcal{L}, V, \delta)$, puis une action d'échelle : 


$$
S=\int_{\delta_{1}}^{\delta_{2}} L(\ln \mathcal{L}, V, \delta) d \delta .
$$

Le principe d'action stationnaire conduit alors à des équations d'EulerLagrange d'échelle :

$$
\frac{d}{d \delta} \frac{\partial \mathscr{L}}{\partial V}=\frac{\partial \mathscr{L}}{\partial \ln \mathcal{L}} .
$$

\subsubsection{Lois d'échelle libres}

La forme la plus simple de ces équations est celle où le deuxième membre s'annule (absence de force d'échelle) et où la fonction de Lagrange prend la forme newtonienne $L \propto V^{2}$. On retrouve là, par cette autre voie, le comportement d' « inertie d'échelle » en loi de puissance. En effet, l'équation de Lagrange s'écrit dans ce cas :

$$
\frac{d V}{d \delta}=0 \Rightarrow V=c s t \text {. }
$$

La constance de $V=\ln (\lambda \varepsilon)$ signifie ici qu'elle est indépendante de $\delta$. L'équation (31) s'intègre alors suivant la forme usuelle $\mathcal{L}=\mathcal{L}_{0}(\lambda \varepsilon)^{\delta}$.

Mais l'avantage principal de cette représentation est qu'elle permet de passer à l'ordre suivant, c'est-à-dire à des comportements non-linéaires de dynamique d'échelle. Identifier la résolution à une vitesse d'échelle, $V=\ln (\lambda \varepsilon)$, conduit naturellement à l'ordre suivant à définir une accélération d'échelle,

$$
\Gamma=d^{2} \ln \mathcal{L} / d \delta^{2}=d \ln (\lambda \varepsilon) / d \delta .
$$

L'introduction d'une force d'échelle permet alors d'écrire une version appliquée aux lois d'échelle de l'équation de la dynamique de Newton (qui n'est rien d'autre que l'équation de Lagrange (33)) :

$$
F=\mu \mathbb{\Gamma}=\mu \frac{d^{2} \ln \mathcal{L}}{d \delta^{2}},
$$

où $\mu$ est une «masse d'échelle », qui mesure comment le système résiste à la force d'échelle.

\subsubsection{Force d'échelle constante}

Considérons tout d'abord le cas d'une force constante. Celle-ci dérive d'un « potentiel d'échelle » $\varphi=\mathbb{F} \ln \mathcal{L}$. On peut écrire l'équation (36) sous la forme

$$
\frac{d^{2} \ln \mathcal{L}}{d \delta^{2}}=G,
$$

où $G=\mathbb{F} / \mu=$ cst. Il s'agit là de l'équivalent, pour les échelles, de ce qu'est la chute des corps en gravité constante. Sa solution est un comportement parabolique : 


$$
V=V_{0}+G \delta ; \ln \mathcal{L}=\ln \mathcal{L}_{0}+V_{0} \delta+\frac{1}{2} G \delta^{2} .
$$

Sous cette forme, la signification physique de ce résultat n'est pas claire. En effet, du point de vue expérimental, c'est en fonction de la résolution que s'observent les variations de $\ln \mathcal{L}$ et éventuellement $\delta$. Après redéfinition des constantes d'intégration, cette solution se réexprime sous la forme :

$$
\delta=\delta_{0}+\frac{1}{G} \ln \left(\frac{\lambda}{\varepsilon}\right) \quad ; \quad \ln \frac{\mathcal{L}}{\mathcal{L}_{0}}=\frac{1}{2 G} \ln ^{2}\left(\frac{\lambda}{\varepsilon}\right) .
$$

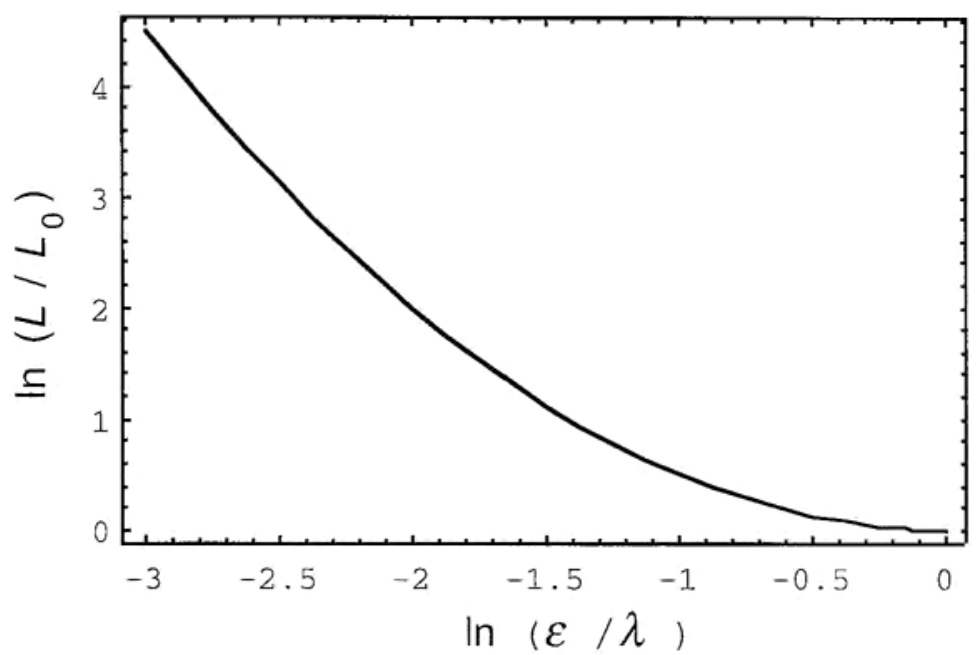

Fig. 4. - Variation de la longueur d'une courbe fractale généralisée en fonction de la résolution, dans le cas d'une force d'échelle constante.

Ainsi la dimension fractale, ordinairement constante, est devenue une fonction linéaire de la log-résolution, et le logarithme de la longueur varie maintenant, non plus linéairement, mais de manière parabolique (voir figure 4 , ci-dessus). Ce résultat est potentiellement applicable à de nombreuses situations, dans tous les domaines où prévaut l'analyse fractale (physique, chimie, biologie et médecine). En effet, il est fréquent qu'après analyse soigneuse de la dépendance d'échelle d'une grandeur, le modèle en loi de puissance soit rejeté pour cause de variation de la pente dans le plan $(\log \mathcal{L}, \log \varepsilon)$. Dans de tels cas, la conclusion selon laquelle le phénomène considéré n'est pas fractal pourrait s'avérer prématurée. Il pourrait au contraire s'agir d'un comportement fractal non-linéaire relevant d'une dynamique d'échelle, auquel cas l'identification et l'étude de la force 
d'échelle responsable de la distorsion pourraient se révéler du plus haut intérêt.

\subsubsection{Oscillateur harmonique d'échelle}

Un autre cas intéressant de potentiel d'échelle est celui de l'oscillateur harmonique. Dans le cas où il est «attractif », l'équation d'échelle s'écrit :

$$
\ln \mathcal{L}^{\prime \prime}+\alpha^{2} \ln \mathcal{L}=0,
$$

où "' désigne la dérivée seconde par rapport à la variable $\delta$. En posant $\alpha=\ln (\lambda \Lambda)$, on trouve que la solution s'écrit :

$$
\ln \mathcal{L}=\left[1-\ln ^{2}(\lambda \varepsilon) / \ln ^{2}(\lambda \Lambda)\right]^{1 / 2} .
$$

Il apparaît ainsi une échelle minimale ou maximale $\Lambda$ pour le système considéré, tandis que la pente $\mathrm{d} \ln \mathcal{L} / \mathrm{d} \ln \varepsilon$ (à ne plus confondre avec le « djinn » $\delta$ dans cette situation non-linéaire) varie entre zéro et l'infini dans le domaine de résolutions permis entre $\lambda$ et $\Lambda$.

Plus intéressant encore est le cas « répulsif », correspondant à un potentiel qu'on peut écrire sous la forme $\varphi=-\left(\ln \mathcal{L} / \delta_{0}\right)^{2} / 2$. La solution s'écrit :

$$
\ln \frac{\mathcal{L}}{\mathcal{L}_{0}}=\delta_{0} \sqrt{\ln ^{2}(\lambda / \varepsilon)-\delta_{0}^{-2}}
$$

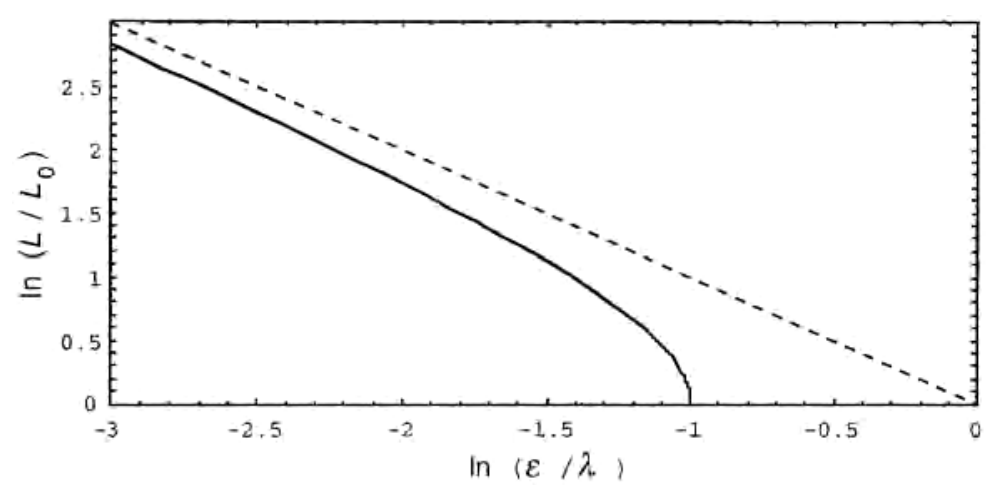

Fig. 5. - Dépendance en fonction de la résolution de la longueur d'une courbe fractale généralisée, solution d'une équation de la dynamique d'échelle dans laquelle le potentiel est celui d'un oscillateur harmonique.

L'intérêt de cette solution (voir figure 5, ci-dessus) est qu'elle redonne comme comportement asymptotique des très grandes ou très petites 
échelles $(\varepsilon<<\lambda$ ou $\varepsilon>>\lambda)$ la solution usuelle $\mathcal{L}=\mathcal{L}_{0}(\lambda / \varepsilon)^{\delta}$ o, de dimension fractale constante $D=1+\delta_{0}$. Par contre, ce comportement rencontre des distorsions croissantes quand la résolution se rapproche d'une échelle maximale $\lambda \mathrm{e}^{-\delta}$ o (ou minimale $\lambda \mathrm{e}^{-\delta}$ o), pour lesquelles la pente (qu'on peut identifier à une dimension fractale effective moins la dimension topologique) devient infinie. En physique, nous avons suggéré qu'un tel comportement pourrait apporter un éclairage nouveau sur le confinement des quarks : en effet, dans le cadre de la réinterprétation des symétries de jauge comme symétries sur les résolutions spatio-temporelles, le groupe de jauge de la chromodynamique quantique est $\mathrm{SU}(3)$, qui est précisément le groupe de symétrie dynamique de l'oscillateur harmonique.

Des solutions de ce type pourraient également présenter de l'intérêt dans le domaine biologique, car on peut interpréter l'existence d'une échelle maximale où la dimension fractale effective devient infinie comme celle d'une paroi, ce qui pourrait fournir des modèles, par exemple, de parois cellulaires. À des échelles plus petites (pour les petits constituants qui évoluent à l'intérieur du système considéré), on tend soit vers l'indépendance d'échelle (pente nulle) dans le premier cas, soit vers le comportement fractal « libre » à pente constante dans le deuxième, ce qui est là encore en accord avec cette interprétation.

Nous avons montré et illustré sur un exemple simple que, dans la nouvelle approche fondée sur la fractalité et la non-différentiabilité, la dynamique de type classique se transformait en une dynamique nouvelle ayant un caractère quasiquantique, naturellement capable de morphogenèse. En effet, on trouve que les particules-tests auxquelles on applique cette méthode constituent des structures finies qui, à une échelle grande devant leur taille caractéristique, pourront elle-mêmes être traitées comme particules. On est ainsi ramené au problème précédent (mais en tenant compte du fait que l'unité naturelle d'échelle, et éventuellement les conditions aux limites et le potentiel ont pu varier), et la même méthode peut alors être appliquée. Une hiérarchie de structures se met ainsi en place, qui combinera des propriétés universelles invariantes d'échelle avec d'autres propriétés spécifiques de l'échelle (relative) particulière considérée. Cette méthode a permis de comprendre des structures observées dans le domaine de l'astrophysique et d'en prédire de nouvelles. Il est probable qu'elle pourrait être transposée dans d'autres parties de la physique, et peut-être dans d'autres disciplines où se pose de manière cruciale les problèmes de morphogenèse (l'exemple de l'halieutique a été proposé ${ }^{30}$ ).

30. Notalle, 2000. 
Un autre développement de l'approche relativiste d'échelle potentiellement applicable hors du champ de la physique est celui qui concerne l'introduction de lois d'échelle généralisées. Nous avons montré comment des lois log-périodiques apparaissaient naturellement dans cette approche quand on passe à des transformations non-linéaires. De telles lois ont été récemment appliquées avec succès à la description de phénomènes aussi divers que les tremblements de terre, les krachs boursiers, l'évolution des espèces ou la chronologie des grandes crises économiques des sociétés humaines. Pour finir, nous avons donné d'autres exemples de lois issues d'une «dynamique d'échelle» qui pourraient également fournir des modèles applicables à des systèmes biologiques.

Laurent NotTALE

(février 2000).

\section{LISTE DES RÉFÉRENCES}

Bez (Nicolas), 2000, in Gascuel, Chavance, Bez et Biseau, éd., 2000, p.17.

Chaline (Jean), Nottale (Laurent) et Grou (Pierre), 1999, «L'arbre de la vie a-t-il une structure fractale?», Comptes rendus de l'Académie des sciences, Paris 328, p. 717.

Dubrulle (Bérangère), Graner (François) et Sornette (Didier), éd., 1997, Scale invariance and beyond. Proceedings of Les Houches school, Paris/Berlin, EDP Sciences/Springer.

EINSTEIN (Albert), 1916, « The foundation of the general theory of relativity », Annalen der Physik, vol. XLIX, p. 769, repr. in Albert EinsteIn, Hendrik A. LoRentz, Hermann Weyl et Hermann Minkowski, The Principle of relativity, trad. angl., New York, Dover publications, 1952, p. 109-164.

El Naschie (Mohamed S.), 1995, in Mohamed S. El NAschie, Otto E. Rössler et Ilya Prigogine, éd., Quantum mechanics, diffusion and chaotic fractals, Oxford, Pergamon, p. 93, 185 et 191.

Gascuel (Didier), Chavance (Pascal), Bez (Nicolas) et Biseau (Alain), éd., 2000, Les Espaces de l'halieutique. Actes du IV forum de l'Halieutique, Rennes, juin 1999, Rennes, IRD Éditions.

Gould (Stephen J.) et Eldredge (N.), 1977, « Punctuated equilibrics. The tempo and mode of evolution reconsidered », Paleobiology, vol. III, 2, p. 115-151.

Grou (Pierre), 1987, L'Aventure économique, Paris, L'Harmattan.

Grou (Pierre), Nottale (Laurent) et Chaline (Jean), 2001, en préparation.

Hermann (Raphaël), 1997, " Numerical simulation of a quantum particle in a box », Journal of Physics, A 30, p. 3967.

Kolwankar (Koran M.) et Gangal (Anil D.), 1998, «Local fractional FokkerPlanck equation », Physicale. Review Letters, vol. LXXX, p. 214.

Landau (Lev) et Lifchitz (Evgueni), 1970, Théorie des champs, Moscou, Mir, p. 368 . 
Mandelbrot (Benoît), 1975, Les Objets fractals, Paris, Flammarion.

Mandelbrot (B.), 1982, The Fractal Geometry of nature, San Francisco, CA, Freeman.

Mandelbrot (B.) et Van Ness (J.W.), 1968, « Fractional Brownian motions, fractional noises and applications », SIAM Review, 10, p. 422.

NotTale (Laurent), 1989, « Fractals and the quantum theory of space-time », International Journal of Modern Physics, A4, p. 5047-5117.

Nottale (L.), 1992, "The theory of scale relativity », International Journal of Modern Physics, A7, p. 4899-4936.

Nottale (L.), 1993, Fractal space-time and microphysics. Towards a theory of scale relativity, Londres, World Scientific.

Nottale (L.), 1995, «Scale relativity. From quantum mechanics to chaotic dynamics », Chaos, Solitons and Fractals, vol. VI, p. 399-410.

NotTALE (L.), 1996a, « Scale relativity and fractal space-time. Applications to quantum physics, cosmology and chaotic systems », Chaos, Solitons and Fractals, vol. VII, p. 877.

NotTale (L.), 1996b, « Scale relativity and quantization of extrasolar planetary systems », Astronomy and Astrophysics, 315, L9.

NotTale (L.), 1997a, «Scale relativity and quantization of the universe. I. Theoretical framework », Astronomy and Astrophysics, 327, p. 867.

Nottale (L.), 1997b, « Scale relativity », in Dubrulle, Graner et Sornette, éd., 1997, p. 249.

Nottale (L.), 1998, La Relativité dans tous ses états. Du mouvement aux changements d'échelle, Paris, Hachette Littératures.

Nottale (L.), 2000, «La théorie de la relativité d'échelle. Réflexions pour une application à l'halieutique », in Gascuel, Chavance, Bez et Biseau, éd., 2000, p. 41-54.

Nottale (Laurent), Chaline (Jean) et Grou (Pierre), 2000, Les Arbres de l'évolution, Paris, Hachette Littératures.

NotTale (Laurent) et SChNeIDER (Jean), 1984, «Fractals and non-standard analysis », Journal of Mathematical Physics, vol. XXV, p. 1296-1300.

Nottale (Laurent), Schumacher (Gérard) et Gay (Jean), 1997, « Scale relativity and quantization of the solar system », Astronomy and Astrophysics, 322, p. 1018.

Nottale (Laurent), Schumacher (Gérard) et Lefèvre (Éric), 2000, « Scale relativity and quantization of exoplanet orbital semi-major axes », Astronomy and Astrophysics, 361, p. 379.

ORD (Garnet), 1983, « Fractal space-time. A geometric analogue of relativistic quantum mechanics », Journal of Physics. A : Mathematical and General, vol. XVI, p. $1869-1884$.

POCHEAU (Alain), 1997, «From scale-invariance to scale-covariance in out of equilibrium systems », in Dubrulle, Graner et Sornette, éd., 1997, p. 209.

Schumacher (Gérard) et NotTale (Laurent), 2001, en préparation.

Sornette (Didier), 1997, « Discrete scale invariance », in Dubrulle, Graner et SorNETTE, éd., 1997, p. 233.

SoRnEtTE (Didier), 1998, «Discrete scale invariance and complex dimensions », Physics Reports, 297, p. 239. 